\title{
Erratum: Spin-Orbit Coupling, Quantum Dots, and Qubits in Monolayer Transition Metal Dichalcogenides [Phys. Rev. X 4, 011034 (2014)]
}

\author{
Andor Kormányos, ${ }^{*}$ Viktor Zólyomi, Neil D. Drummond, and Guido Burkard
}

(Received 19 May 2014; published 22 July 2014)

DOI: 10.1103/PhysRevX.4.039901

Subject Areas: Condensed Matter Physics, Nanophysics,

Spintronics

In our paper, there are misprints in Table II regarding the values of $g_{v l}$ and $\tilde{g}_{v l}$ that need to be corrected. In addition, we have decided that in order to adhere to the standard definitions used in the literature regarding Hamiltonians that involve $g$ factor(s), a minor change is appropriate. We redefine $\tilde{g}_{v l}$ in Eq. (2): $\tilde{g}_{v l} \rightarrow-\tilde{g}_{v l}$, and therefore Eq. (2) should read

$$
\tilde{H}_{e l}^{\tau, s}+\tilde{H}_{v l}^{\tau}+\tilde{H}_{s p}^{s}=\frac{\hbar^{2} \hat{q}_{+} \hat{q}_{-}}{2 m_{\mathrm{eff}}^{\tau, s}}+\frac{1+\tau}{2} \operatorname{sgn}\left(B_{z}\right) \hbar \omega_{c}^{\tau, s}+\frac{\tau}{2} \tilde{g}_{v l} \mu_{B} B_{z}+\frac{1}{2} \mu_{B} g_{s o}^{\perp} s_{z} B_{z},
$$

where $\hbar \omega_{c}^{\tau, s}=e\left|B_{z}\right| / m_{\text {eff }}^{\tau, s}$. Accordingly, the second sentence below Eq. (2) should read as follows: "The next term $\tilde{H}_{v l}^{\tau}=\frac{\tau}{2} \tilde{g}_{v l} \mu_{B} B_{z}$ breaks the valley symmetry of Landau levels."

The $\tilde{g}_{v l}$ values shown in the first row of Table II of the manuscript need to be corrected because they are actually half of the correct values. In addition, a minus-sign change comes from the above-mentioned redefinition of $\tilde{g}_{v l}$. Furthermore, since $g_{v l}$ in Eq. (4) is defined through $\tilde{g}_{v l}$, both its definition and its values (shown in the third row of Table II) are also modified. After redefinition, $g_{v l}$ is given by $g_{v l}=\left(2 m_{e} / m_{\mathrm{eff}}^{0}\right)+\tilde{g}_{v l}$. The new Table II of the manuscript, with the corrected values of the valley $g$ factors $\tilde{g}_{v l}$ and $g_{v l}$, is shown below. Note that the spin $g$ factors $g_{s o}^{\perp}$ and $g_{s p}^{\perp}$ do not need to be changed.

Some of the $g_{v l}$ values also appear in the captions of Figs. 5 and 6, which therefore also need to be corrected.

The caption of Fig. 5 should read as follows.

"Spectrum of a 40-nm $\mathrm{WS}_{2}$ quantum dot as a function of the perpendicular magnetic field $B_{z}>0$. Black (red) lines show the spin $\uparrow(\downarrow)$ states from the valley $K\left(K^{\prime}\right)$. The values of $m_{\text {eff }}^{\tau, s}$ can be found in Table I, whereas $g_{v l}=-3.36$ and $g_{s p}^{\perp}=1.99$ (see Table II)."

TABLE II. Valley $\left(\tilde{g}_{v l}, g_{v l}\right)$ and spin $\left(g_{s o}^{\perp}, g_{s p}^{\perp}\right) g$ factors for different transition metal dichalcogenides.

\begin{tabular}{lrrrr}
\hline \hline & $\mathrm{MoS}_{2}$ & $\mathrm{WS}_{2}$ & $\mathrm{MoSe}_{2}$ & $\mathrm{WSe}_{2}$ \\
\hline$\tilde{g}_{v l}$ & -7.14 & -9.92 & -6.06 & -8.68 \\
$\left|g_{s o}^{\perp}\right|$ & 0.21 & 0.84 & 0.29 & 0.87 \\
$g_{v l}^{\perp}$ & -3.36 & -2.71 & -2.85 \\
$g_{s p}^{\perp}$ & -2.83 & 1.99 & 2.07 & 2.04 \\
\hline \hline
\end{tabular}

Similarly, the caption of Fig. 6 needs to be modified, and it should read as follows.

"Spectrum of a 40-nm WS 2 quantum dot as a function of the perpendicular magnetic field $B_{z}>0$. The values of $m_{\mathrm{eff}}^{\tau, s} \mathrm{can}$ be found in Table I, and we use $g_{v l}=-1.94$ and $g_{s p}^{\perp}=1.84$ (see Fig. 5). Black (red) lines show spin $\uparrow(\downarrow)$ states from the $K$ $\left(K^{\prime}\right)$ valley."

Finally, the sentence before Eq. (B7) and the definition of $\tilde{g}_{v l}$ should read as follows.

"The zeroth-order term yields the valley-splitting Hamiltonian $\tilde{H}_{v l}^{\tau}=\frac{\tau}{2} \tilde{g}_{v l} \mu_{B} B_{z}$, with

$$
\tilde{g}_{v l}=-2\left(1+4 m_{e}\left|\tilde{\gamma}_{3}\right|^{2} / E_{b g}\right) \text {." }
$$

We emphasize that the numerical calculations for quantum dots shown in Figs. 4-6 are not affected by these changes. All conclusions of the paper remain unchanged.

\footnotetext{
*andor.kormanyos@uni-konstanz.de
}

Published by the American Physical Society under the terms of the Creative Commons Attribution 3.0 License. Further distribution of this work must maintain attribution to the author(s) and the published articles title, journal citation, and DOI. 